\title{
On the growth measures of entire and meromorphic functions focusing their generalized relative type and generalized relative weak type
}

\author{
Sanjib Kumar Datta \\ Department of Mathematics, \\ University of Kalyani, India \\ email: sanjib_kr_datta@yahoo.co.in
}

\author{
Tanmay Biswas \\ Rajbari, Rabindrapalli, India \\ email: \\ tanmaybiswas_math@rediffmail.com
}

\begin{abstract}
In this paper we study some comparative growth properties of composite entire and meromorphic functions on the basis of their generalized relative order, generalized relative type and generalized relative weak type with respect to another entire function.
\end{abstract}

\section{Introduction}

Let $f$ be an entire function defined in the finite complex plane $\mathbb{C}$. The maximum modulus function corresponding to entire $f$ is defined as $M_{f}(r)=$ $\max \{|f(z)|:|z|=r\}$. If $\mathrm{f}$ is non-constant then it has the following property:

Property (A) [2] A non-constant entire function $f$ is said have the Property (A) if for any $\sigma>1$ and for all sufficiently large values of $r,\left[M_{f}(r)\right]^{2} \leq M_{f}\left(r^{\sigma}\right)$ holds. For examples of functions with or without the Property (A), one may see $[2]$.

2010 Mathematics Subject Classification: 30D20, 30D30, 30D35

Key words and phrases: meromorphic function, entire function, generalized relative order, generalized relative type, generalized relative weak type 
For any two entire functions $f$ and $g$, the ratio $\frac{M_{f}(r)}{M_{g}(r)}$ as $r \rightarrow \infty$ is called the growth of $f$ with respect to $g$ in terms of their maximum moduli. The order (lower order) of an entire function $f$ which is generally used in computational purpose is defined in terms of the growth of $f$ respect to the $\exp z$ function which is as follows:

$$
\begin{gathered}
\rho_{f}=\limsup _{r \rightarrow \infty} \frac{\log \log M_{f}(r)}{\log \log M_{\exp z}(r)}=\limsup _{r \rightarrow \infty} \frac{\log \log M_{f}(r)}{\log (r)} \\
\left(\lambda_{f}=\liminf _{r \rightarrow \infty} \frac{\log \log M_{f}(r)}{\log \log M_{\exp z}(r)}=\liminf _{r \rightarrow \infty} \frac{\log \log M_{f}(r)}{\log (r)}\right) .
\end{gathered}
$$

When $f$ is meromorphic, $M_{f}(r)$ cannot be defined as $f$ is not analytic. In this case one may define another function $T_{f}(r)$ known as Nevanlinna's Characteristic function of $f$, playing the same role as maximum modulus function in the following manner:

$$
T_{f}(r)=N_{f}(r)+m_{f}(r),
$$

where the function $N_{f}(r, a)\left(\bar{N}_{f}(r, a)\right)$ known as counting function of a-points (distinct a-points) of meromorphic $f$ is defined as

$$
\begin{gathered}
N_{f}(r, a)=\int_{0}^{r} \frac{n_{f}(t, a)-n_{f}(0, a)}{t} d t+\bar{n}_{f}(0, a) \log r \\
\left(\bar{N}_{f}(r, a)=\int_{0}^{r} \frac{\bar{n}_{f}(t, a)-\bar{n}_{f}(0, a)}{t} d t+\bar{n}_{f}(0, a) \log r\right),
\end{gathered}
$$

moreover we denote by $n_{f}(r, a)\left(\bar{n}_{f}(r, a)\right)$ the number of a-points (distinct a-points) of $f$ in $|z| \leq r$ and an $\infty$-point is a pole of $f$. In many occasions $N_{f}(r, \infty)$ and $\bar{N}_{f}(r, \infty)$ are denoted by $N_{f}(r)$ and $\bar{N}_{f}(r)$ respectively.

And the function $m_{f}(r, \infty)$ alternatively denoted by $m_{f}(r)$ known as the proximity function of $f$ is defined as follows:

$$
\begin{aligned}
& m_{f}(r)=\frac{1}{2 \pi} \int_{0}^{2 \pi} \log ^{+}\left|f\left(r e^{i \theta}\right)\right| d \theta, \quad \text { where } \\
& \log ^{+} x=\max (\log x, 0) \text { for all } x \geqslant 0 .
\end{aligned}
$$


Also we may denote $m\left(r, \frac{1}{f-a}\right)$ by $m_{f}(r, a)$.

If $f$ is entire function, then the Nevanlinna's Characteristic function $T_{f}(r)$ of $f$ is defined as

$$
T_{f}(r)=m_{f}(r) .
$$

Further, if $f$ is non-constant entire then $T_{f}(r)$ is strictly increasing and continuous functions of $r$. Also its inverse $T_{f}^{-1}:\left(T_{f}(0), \infty\right) \rightarrow(0, \infty)$ exist and is such that $\lim _{s \rightarrow \infty} T_{f}^{-1}(s)=\infty$. Also the ratio $\frac{T_{f}(r)}{T_{g}(r)}$ as $r \rightarrow \infty$ is called the growth of $f$ with respect to $g$ in terms of the Nevanlinna's Characteristic functions of the meromorphic functions $f$ and $g$. Moreover in case of meromorphic functions, the growth indicators such as order and lower order which are classical in complex analysis are defined in terms of their growths with respect to the $\exp z$ function as the following:

$$
\begin{gathered}
\rho_{f}=\limsup _{r \rightarrow \infty} \frac{\log T_{f}(r)}{\log T_{\exp z}(r)}=\limsup _{r \rightarrow \infty} \frac{\log T_{f}(r)}{\log \left(\frac{r}{\pi}\right)}=\limsup _{r \rightarrow \infty} \frac{\log T_{f}(r)}{\log (r)+O(1)} \\
\left(\lambda_{f}=\liminf _{r \rightarrow \infty} \frac{\log T_{f}(r)}{\log T_{\exp z}(r)}=\liminf _{r \rightarrow \infty} \frac{\log T_{f}(r)}{\log \left(\frac{r}{\pi}\right)}=\liminf _{r \rightarrow \infty} \frac{\log T_{f}(r)}{\log (r)+O(1)}\right) .
\end{gathered}
$$

Bernal [1], [2] introduced the relative order between two entire functions to avoid comparing growth just with $\exp z$. Extending the notion of relative order as cited in the reference, Lahiri and Banerjee [9] introduced the definition of relative order of a meromorphic functions with respect to another entire function.

For entire and meromorphic functions, the notion of the growth indicators of its such as generalized order, generalized type and generalized weak type are classical in complex analysis and during the past decades, several researchers have already been continued their studies in the area of comparative growth properties of composite entire and meromorphic functions in different directions using the growth indicator such as generalized order, generalized type and generalized weak type. But at that time, the concept of generalized relative order and consequently generalized relative type and generalized relative weak type of entire and meromorphic function with respect to another entire function which have been discussed in the next section was mostly unknown to complex analysis and was not aware of the technical advantage given by such notion which gives an idea to avoid comparing growth just with exp function to calculate generalized order, generalized type and generalized weak type respectively. Therefore the growth of composite entire and meromorphic functions can be studied on the basis of their generalized relative order, generalized 
relative type and generalized relative weak which has been investigated in this paper.

\section{Notation and preliminary remarks}

We denote by $\mathbb{C}$ the set of all finite complex numbers. Let $f$ be a meromorphic function and $g$ be an entire function defined on $\mathbb{C}$. We use the standard notations and definitions of the theory of entire and meromorphic functions which are available in [8] and [12]. Hence we do not explain those in details. In the consequence we use the following notation:

$$
\log ^{[k]} x=\log \left(\log ^{[k-1]} x\right) \text { for } k=1,2,3, \ldots \text { and } \log ^{[0]} x=x .
$$

Now we just recall some definitions which will be needed in the sequel.

Definition 1 The order $\rho_{f}$ and lower order $\lambda_{f}$ of an entire function $f$ are defined as

$$
\rho_{\mathrm{f}}=\limsup _{\mathrm{r} \rightarrow \infty} \frac{\log ^{[2]} M_{\mathrm{f}}(\mathrm{r})}{\log \mathrm{r}} \text { and } \lambda_{\mathrm{f}}=\liminf _{\mathrm{r} \rightarrow \infty} \frac{\log ^{[2]} \mathrm{M}_{\mathrm{f}}(\mathrm{r})}{\log \mathrm{r}} .
$$

When $\mathrm{f}$ is meromorphic then

$$
\rho_{\mathrm{f}}=\limsup _{\mathrm{r} \rightarrow \infty} \frac{\log \mathrm{T}_{\mathrm{f}}(\mathrm{r})}{\log \mathrm{r}} \text { and } \lambda_{\mathrm{f}}=\liminf _{\mathrm{r} \rightarrow \infty} \frac{\log \mathrm{T}_{\mathrm{f}}(\mathrm{r})}{\log \mathrm{r}} .
$$

In this connection Sato [10] define the generalized order $\rho_{f}^{[l]}$ (respectively, generalized lower order $\lambda_{f}^{[l]}$ ) of an entire function $f$ which is defined as

$$
\rho_{\mathrm{f}}^{[l]}=\limsup _{r \rightarrow \infty} \frac{\log ^{[l]} M_{f}(r)}{\log r}\left(\text { respectively } \lambda_{f}^{[l]}=\liminf _{r \rightarrow \infty} \frac{\log ^{[l]} M_{f}(r)}{\log r}\right)
$$

where $l=1,2,3 \ldots$

For meromorphic $f$, the above definition reduces to

$$
\rho_{\mathrm{f}}^{[l]}=\limsup _{r \rightarrow \infty} \frac{\log ^{[l-1]} \mathrm{T}_{\mathrm{f}}(\mathrm{r})}{\log r}\left(\text { respectively } \lambda_{\mathrm{f}}^{[l]}=\liminf _{\mathrm{r} \rightarrow \infty} \frac{\log ^{[l-1]} \mathrm{T}_{\mathrm{f}}(\mathrm{r})}{\log r}\right)
$$

for any $l \geq 1$.

These definitions extended the definitions of order $\rho_{f}$ and lower order $\lambda_{f}$ of an entire or meromorphic function $f$ which are classical in complex analysis for integer $l=2$ since these correspond to the particular case $\rho_{f}^{[2]}=\rho_{f}(2,1)=\rho_{f}$ and $\lambda_{f}^{[2]}=\lambda_{f}(2,1)=\lambda_{f}$. 
Definition 2 The type $\sigma_{f}$ and lower type $\bar{\sigma}_{f}$ of an entire function $f$ are defined as

$$
\sigma_{f}=\limsup _{r \rightarrow \infty} \frac{\log M_{f}(r)}{r^{\rho_{f}}} \text { and } \bar{\sigma}_{f}=\liminf _{r \rightarrow \infty} \frac{\log M_{f}(r)}{r^{\rho_{f}}}, 0<\rho_{f}<\infty .
$$

If $\mathrm{f}$ is meromorphic then

$$
\sigma_{f}=\limsup _{r \rightarrow \infty} \frac{T_{f}(r)}{r^{\rho_{f}}} \text { and } \bar{\sigma}_{f}=\liminf _{r \rightarrow \infty} \frac{T_{f}(r)}{r^{\rho_{f}}}, \quad 0<\rho_{f}<\infty .
$$

Consequently the generalized type $\sigma_{f}^{[l]}$ and generalized lower type $\bar{\sigma}_{f}^{[l]}$ of an entire function $f$ are defined as

$$
\sigma_{f}^{[l]}=\limsup _{r \rightarrow \infty} \frac{\log ^{[l-1]} M_{f}(r)}{r_{f}^{[l]}} \text { and } \bar{\sigma}_{f}^{[l]}=\liminf _{r \rightarrow \infty} \frac{\log ^{[l-1]} M_{f}(r)}{r_{f}^{[l]}}, 0<\rho_{f}^{[l]}<\infty
$$

where $l \geq 1$. If $f$ is meromorphic then

$$
\sigma_{f}^{[l]}=\limsup _{r \rightarrow \infty} \frac{\log ^{[l-2]} T_{f}(r)}{r^{\rho_{f}^{[l]}}} \text { and } \bar{\sigma}_{f}^{[l]}=\liminf _{r \rightarrow \infty} \frac{\log ^{[l-2]} T_{f}(r)}{r^{\rho_{f}^{[l]}}}, 0<\rho_{f}^{[l]}<\infty
$$

where $l \geq 1$. Moreover, when $l=2$ then $\sigma_{f}^{[2]}$ and $\bar{\sigma}_{f}^{[2]}$ are correspondingly denoted as $\sigma_{f}$ and $\bar{\sigma}_{f}$ which are respectively known as type and lower type of entire or meromorphic $f$.

Datta and Jha [6] introduced the definition of weak type of an entire function of finite positive lower order in the following way:

Definition 3 [6] The weak type $\tau_{f}$ and the growth indicator $\tau_{f}$ of an entire function $\mathrm{f}$ of finite positive lower order $\lambda_{\mathrm{f}}$ are defined by

$$
\bar{\tau}_{f}=\limsup _{r \rightarrow \infty} \frac{\log M_{f}(r)}{r^{\lambda_{f}}} \text { and } \tau_{f}=\liminf _{r \rightarrow \infty} \frac{\log M_{f}(r)}{r^{\lambda_{f}}}, 0<\lambda_{f}<\infty .
$$

When $\mathrm{f}$ is meromorphic then

$$
\bar{\tau}_{f}=\limsup _{r \rightarrow \infty} \frac{T_{f}(r)}{r^{\lambda_{f}}} \text { and } \tau_{f}=\liminf _{r \rightarrow \infty} \frac{T_{f}(r)}{r^{\lambda_{f}}}, 0<\lambda_{f}<\infty .
$$

Similarly, extending the notion of weak type as introduced by Datta and Jha [6], one can define generalized weak type to determine the relative growth of two entire functions having same non zero finite generalized lower order in the following manner: 
Definition 4 The generalized weak type $\tau_{\mathrm{f}}^{[\mathrm{l]}}$ for $\mathrm{l} \geq 1$ of an entire function $\mathrm{f}$ of finite positive generalized lower order $\lambda_{\mathrm{f}}^{[\mathrm{ll}}$ are defined by

$$
\tau_{f}^{[l]}=\liminf _{r \rightarrow \infty} \frac{\log ^{[l-1]} M_{f}(r)}{r_{f}^{[l]}}, 0<\lambda_{f}^{[l]}<\infty .
$$

Also one may define the growth indicator $\bar{\tau}_{\mathrm{f}}^{[\mathrm{l}]}$ of an entire function $\mathrm{f}$ in the following way:

$$
\bar{\tau}_{f}^{[l]}=\limsup _{r \rightarrow \infty} \frac{\log ^{[l-1]} M_{f}(r)}{r_{f}^{\lambda_{f}^{[l]}}}, 0<\lambda_{f}^{[l]}<\infty .
$$

When $\mathrm{f}$ is meromorphic then

$$
\tau_{f}^{[l]}=\liminf _{r \rightarrow \infty} \frac{\log ^{[l-2]} T_{f}(r)}{r_{f}^{\lambda_{f}^{[l]}}} \text { and } \bar{\tau}_{f}^{[l]}=\limsup _{r \rightarrow \infty} \frac{\log ^{[l-2]} T_{f}(r)}{r^{\lambda_{f}^{[l]}}}, 0<\lambda_{f}^{[l]}<\infty .
$$

If an entire function $g$ is non-constant then $M_{g}(r)$ and $T_{g}(r)$ are both strictly increasing and continuous function of $r$. Hence there exists inverse functions $M_{g}^{-1}:(|f(0)|, \infty) \rightarrow(0, \infty)$ with $\lim _{s \rightarrow \infty} M_{g}^{-1}(s)=\infty$ and $T_{g}^{-1}:\left(T_{g}(0), \infty\right) \rightarrow$ $(0, \infty)$ with $\lim _{s \rightarrow \infty} \mathrm{T}_{g}^{-1}(s)=\infty$ respectively.

Bernal [1], [2] introduced the definition of relative order of af an entire function $f$ with respect to an entire function $g$, denoted by $\rho_{g}(f)$ as follows:

$$
\begin{aligned}
\rho_{g}(f) & =\inf \left\{\mu>0: M_{f}(r)<M_{g}\left(r^{\mu}\right) \text { for all } r>r_{0}(\mu)>0\right\} \\
& =\limsup _{r \rightarrow \infty} \frac{\log M_{g}^{-1} M_{f}(r)}{\log r} .
\end{aligned}
$$

The definition coincides with the classical one [11] if $g(z)=\exp z$.

Similarly, one can define the relative lower order of an entire function $f$ with respect to an entire function $g$ denoted by $\lambda_{g}(f)$ as follows:

$$
\lambda_{g}(f)=\liminf _{r \rightarrow \infty} \frac{\log M_{g}^{-1} M_{f}(r)}{\log r} .
$$

Extending this notion, Lahiri and Banerjee [9] introduced the definition of relative order of a meromorphic function $f$ with respect to an entire function $g$, denoted by $\rho_{g}(f)$ as follows:

$$
\begin{aligned}
\rho_{g}(f) & =\inf \left\{\mu>0: T_{f}(r)<T_{g}\left(r^{\mu}\right) \text { for all sufficiently large } r\right\} \\
& =\limsup _{r \rightarrow \infty} \frac{\log T_{g}^{-1} T_{f}(r)}{\log r} .
\end{aligned}
$$


The definition coincides with the classical one [9] if $g(z)=\exp z$.

In the same way, one can define the relative lower order of a meromorphic function $f$ with respect to an entire $g$ denoted by $\lambda_{g}(f)$ in the following manner:

$$
\lambda_{g}(f)=\liminf _{r \rightarrow \infty} \frac{\log T_{g}^{-1} T_{f}(r)}{\log r} .
$$

Further, Banerjee and Jana [6] gave a more generalized concept of relative order of a meromorphic function with respect to an entire function in the following way:

Definition 5 [6] If $l \geq 1$ is a positive integer, then the $l$ - th generalized relative order of a meromorphic function $\mathrm{f}$ with respect to an entire function $\mathrm{g}$, denoted by $\rho_{\mathrm{g}}^{[\mathrm{ll}]}(\mathrm{f})$ is defined by

$$
\rho_{g}^{[l]}(f)=\limsup _{r \rightarrow \infty} \frac{\log ^{[l]} \mathrm{T}_{\mathrm{g}}^{-1} \mathrm{~T}_{\mathrm{f}}(\mathrm{r})}{\log \mathrm{r}} .
$$

Likewise one can define the generalized relative lower order of a meromorphic function $\mathrm{f}$ with respect to an entire function $\mathrm{g}$ denoted by $\lambda_{\mathrm{g}}^{[\mathrm{l]}}(\mathrm{f})$ as

$$
\lambda_{g}^{[l]}(f)=\liminf _{r \rightarrow \infty} \frac{\log ^{[l]} T_{g}^{-1} T_{f}(r)}{\log r} .
$$

In the case of meromorphic functions, it therefore seems reasonable to define suitably the generalized relative type and generalized relative weak type of a meromorphic function with respect to an entire function to determine the relative growth of two meromorphic functions having same non zero finite generalized relative order or generalized relative lower order with respect to an entire function. Next we give such definitions of generalized relative type and generalized relative weak type of a meromorphic function $f$ with respect to an entire function $\mathrm{g}$ which are as follows:

Definition 6 The generalized relative type $\sigma_{g}^{[l]}(\mathbf{f})$ of a meromorphic function $f$ with respect to an entire function $\mathrm{g}$ are defined as

$$
\sigma_{g}^{[l]}(f)=\limsup _{r \rightarrow \infty} \frac{\log ^{[l-1]} T_{g}^{-1} T_{f}(r)}{r_{g}^{[l]}(f)}, \text { where } 0<\rho_{g}^{[[l]]}(f)<\infty .
$$

Similarly, one can define the generalized lower relative type $\bar{\sigma}_{g}(f)$ in the following way:

$$
\bar{\sigma}_{g}^{[l]}(f)=\liminf _{r \rightarrow \infty} \frac{\log ^{[l-1]} T_{g}^{-1} T_{f}(r)}{r_{g}^{[l]}(f)} \text {, where } 0<\rho_{g}^{[l]}(f)<\infty .
$$


Definition 7 The generalized relative weak type $\tau_{g}^{[l]}(\mathrm{f})$ of a meromorphic function $\mathrm{f}$ with respect to an entire function $\mathrm{g}$ with finite positive relative lower order $\lambda_{g}^{[l]}(\mathrm{f})$ is defined by

$$
\tau_{g}^{[l]}(f)=\liminf _{r \rightarrow \infty} \frac{\log ^{[l-1]} T_{g}^{-1} T_{f}(r)}{r_{g}^{\lambda_{g}^{[l]}(f)}} .
$$

In a like manner, one can define the growth indicator $\bar{\tau}_{g}^{[l]}(f)$ of a meromorphic function $\mathrm{f}$ with respect to an entire function $\mathrm{g}$ with finite positive relative lower order $\lambda_{\mathrm{g}}^{[\mathrm{ll}]}(\mathrm{f})$ as

$$
\bar{\tau}_{g}^{[l]}(f)=\limsup _{r \rightarrow \infty} \frac{\log ^{[l-1]} T_{g}^{-1} T_{f}(r)}{r^{\lambda_{g}^{[l]}}(f)} .
$$

\section{Lemmas}

In this section we present some lemmas which will be needed in the sequel.

Lemma 1 [3] Let $\mathrm{f}$ be meromorphic and $\mathrm{g}$ be entire then for all sufficiently large values of $r$,

$$
\mathrm{T}_{\mathrm{f} \circ \mathrm{g}}(\mathrm{r}) \leqslant\{1+\mathrm{o}(1)\} \frac{\mathrm{T}_{\mathrm{g}}(\mathrm{r})}{\log \mathrm{M}_{\mathrm{g}}(\mathrm{r})} \mathrm{T}_{\mathrm{f}}\left(\mathrm{M}_{\mathrm{g}}(\mathrm{r})\right) .
$$

Lemma 2 [4] Let $\mathrm{f}$ be meromorphic and $\mathrm{g}$ be entire and suppose that $0<\mu<$ $\rho_{\mathrm{g}} \leq \infty$. Then for a sequence of values of $\mathrm{r}$ tending to infinity,

$$
\mathrm{T}_{\text {fog }}(\mathrm{r}) \geq \mathrm{T}_{\mathrm{f}}\left(\exp \left(\mathrm{r}^{\mu}\right)\right) \text {. }
$$

Lemma 3 [7] Let $\mathrm{f}$ be an entire function which satisfy the Property $(A), \beta>$ $0, \delta>1$ and $\alpha>2$. Then

$$
\beta T_{f}(r)<T_{f}\left(\alpha r^{\delta}\right)
$$

\section{Main results}

In this section we present the main results of the paper.

Theorem 1 Let $\mathrm{f}$ be meromorphic, $\mathrm{g}$ and $\mathrm{h}$ be any two entire functions such that $0<\lambda_{\mathrm{h}}^{[\mathrm{l}]}(\mathrm{f}) \leq \rho_{\mathrm{h}}^{[\mathrm{l}]}(\mathrm{f})<\infty, \sigma_{\mathrm{g}}<\infty$ and $\mathrm{h}$ satisfy the Property (A) where $l>1$. Then

$$
\limsup \frac{\log ^{[l]} \mathrm{T}_{h}^{-1} \mathrm{~T}_{\mathrm{fog}}(\mathrm{r})}{\log ^{[l]} \mathrm{T}_{h}^{-1} \mathrm{~T}_{\mathrm{f}}\left(\exp \mathrm{r}^{\left.\rho_{g}\right)}\right.} \leq \frac{\sigma_{g} \cdot \rho_{h}^{[l]}(f)}{\lambda_{h}^{[l]}(f)} .
$$


Proof. Let us suppose that $\alpha>2$.

Since $T_{h}^{-1}(r)$ is an increasing function $r$, it follows from Lemma 1, Lemma 3 and the inequality $T_{g}(r) \leq \log M_{g}(r)\{c f .[8]\}$ that for all sufficiently large values of $r$ we have

$$
\begin{aligned}
& \mathrm{T}_{\mathrm{h}}^{-1} \mathrm{~T}_{\mathrm{f \circ g}}(\mathrm{r}) \leqslant \mathrm{T}_{\mathrm{h}}^{-1}\left[\{1+\mathrm{o}(1)\} \mathrm{T}_{\mathrm{f}}\left(\mathrm{M}_{\mathrm{g}}(\mathrm{r})\right)\right] \\
& \text { i.e., } T_{h}^{-1} T_{f \circ g}(r) \leqslant \alpha\left[T_{h}^{-1} T_{f}\left(M_{g}(r)\right)\right]^{\delta} \\
& \text { i.e., } \log ^{[l]} \mathrm{T}_{h}^{-1} \mathrm{~T}_{\text {fog }}(r) \leqslant \log ^{[l]} \mathrm{T}_{h}^{-1} \mathrm{~T}_{f}\left(M_{g}(r)\right)+\mathrm{O}(1) \\
& \text { i.e., } \frac{\log ^{[l]} T_{h}^{-1} T_{f \circ g}(r)}{\log ^{[l]} T_{h}^{-1} T_{f}\left(\exp r^{\rho_{g}}\right)} \\
& \leq \frac{\log ^{[l]} \mathrm{T}_{h}^{-1} \mathrm{~T}_{f}\left(M_{g}(r)\right)+\mathrm{O}(1)}{\log ^{[l]} \mathrm{T}_{h}^{-1} \mathrm{~T}_{\mathrm{f}}\left(\exp r^{\rho_{g}}\right)}=\frac{\log ^{[l]} \mathrm{T}_{h}^{-1} \mathrm{~T}_{\mathrm{f}}\left(M_{g}(\mathrm{r})\right)+\mathrm{O}(1)}{\log M_{g}(\mathrm{r})} \\
& \frac{\log M_{g}(r)}{r^{\rho_{g}}} \cdot \frac{\log \exp r^{\rho_{g}}}{\log ^{[l]} T_{h}^{-1} T_{f}\left(\exp r^{\rho_{g}}\right)} \\
& \text { i.e., } \limsup _{r \rightarrow \infty} \frac{\log ^{[l]} \mathrm{T}_{h}^{-1} \mathrm{~T}_{\text {fog }}(\mathrm{r})}{\log ^{[l]} \mathrm{T}_{h}^{-1} \mathrm{~T}_{f}\left(\exp r^{\rho_{g}}\right)} \\
& \leq \limsup _{r \rightarrow \infty} \frac{\log ^{[l]} \mathrm{T}_{h}^{-1} \mathrm{~T}_{f}\left(M_{g}(r)\right)+\mathrm{O}(1)}{\log M_{g}(\mathrm{r})} \cdot \limsup _{r \rightarrow \infty} \frac{\log M_{g}(r)}{r^{\rho_{g}}} \text {. } \\
& \limsup _{r \rightarrow \infty} \frac{\log \exp r^{\rho_{g}}}{\log ^{[l]} T_{h}^{-1} T_{f}\left(\exp r^{\rho_{g}}\right)} \\
& \text { i.e., } \limsup _{r \rightarrow \infty} \frac{\log ^{[l]} T_{h}^{-1} T_{f \circ g}(r)}{\log ^{[l]} T_{h}^{-1} T_{f}\left(\exp r^{\rho_{g}}\right)} \leq \rho_{h}^{[l]}(f) \cdot \sigma_{g} \cdot \frac{1}{\lambda_{h}^{[l]}(f)} \text {. }
\end{aligned}
$$

Thus the theorem is established.

In the line of Theorem 1 the following theorem can be proved:

Theorem 2 Let $\mathrm{f}$ be a meromorphic function, $\mathrm{g}$ and $\mathrm{h}$ be any two entire functions such that $\lambda_{\mathrm{h}}^{[\mathrm{ll}}(\mathrm{g})>0, \rho_{\mathrm{h}}^{[\mathrm{ll}]}(\mathrm{f})<\infty, \sigma_{\mathrm{g}}<\infty$ and $\mathrm{h}$ satisfy the Property (A) where $\mathrm{l}>1$. Then

$$
\limsup \frac{\log ^{[l]} \mathrm{T}_{h}^{-1} \mathrm{~T}_{\text {fog }}(\mathrm{r})}{\log ^{[l]} \mathrm{T}_{h}^{-1} \mathrm{~T}_{g}\left(\exp r^{\left.\rho_{g}\right)}\right.} \leq \frac{\sigma_{g} \cdot \rho_{h}^{[l]}(f)}{\lambda_{h}^{[l]}(g)} .
$$


Using the notion of lower type we may state the following two theorems without proof because it can be carried out in the line of Theorem 1 and Theorem 2 respectively.

Theorem 3 Let $\mathrm{f}$ be meromorphic, $\mathrm{g}$ and $\mathrm{h}$ be any two entire functions such that $0<\lambda_{h}^{[l]}(\mathrm{f}) \leq \rho_{\mathrm{h}}^{[\mathrm{l]}}(\mathrm{f})<\infty, \bar{\sigma}_{\mathrm{g}}<\infty$ and $\mathrm{h}$ satisfy the Property (A) where $l>1$. Then

$$
\liminf \frac{\log ^{[l]} \mathrm{T}_{h}^{-1} \mathrm{~T}_{\text {fog }}(\mathrm{r})}{\log ^{[l]} \mathrm{T}_{h}^{-1} \mathrm{~T}_{\mathrm{f}}\left(\exp \mathrm{r}^{\rho_{g}}\right)} \leq \frac{\bar{\sigma}_{g} \cdot \rho_{h}^{[l]}(f)}{\lambda_{h}^{[l]}(f)} .
$$

Theorem 4 Let $\mathrm{f}$ be a meromorphic function, $\mathrm{g}$ and $\mathrm{h}$ be any two entire functions such that $\lambda_{\mathrm{h}}^{[\mathrm{ll}}(\mathrm{g})>0, \rho_{\mathrm{h}}^{[\mathrm{l}]}(\mathrm{f})<\infty, \bar{\sigma}_{\mathrm{g}}<\infty$ and $\mathrm{h}$ satisfy the Property (A) where $\mathrm{l}>1$. Then

$$
\liminf \frac{\log ^{[l]} \mathrm{T}_{h}^{-1} \mathrm{~T}_{f \circ g}(\mathrm{r})}{\log ^{[l]} \mathrm{T}_{h}^{-1} \mathrm{~T}_{g}\left(\exp \mathrm{r}^{\left.\rho_{g}\right)}\right.} \leq \frac{\bar{\sigma}_{g} \cdot \rho_{h}^{[l]}(f)}{\lambda_{h}^{[l]}(g)} .
$$

Using the concept of the growth indicators $\tau_{g}$ and $\bar{\tau}_{g}$ of an entire function $g$, we may state the subsequent four theorems without their proofs since those can be carried out in the line of Theorem 1, Theorem 2, Theorem 3 and Theorem 4 respectively.

Theorem 5 Let $\mathrm{f}$ be meromorphic, $\mathrm{g}$ and $\mathrm{h}$ be any two entire functions such that $0<\lambda_{\mathrm{h}}^{[\mathrm{l}]}(\mathrm{f}) \leq \rho_{\mathrm{h}}^{[\mathrm{l}]}(\mathrm{f})<\infty, \bar{\tau}_{\mathrm{g}}<\infty$ and $\mathrm{h}$ satisfy the Property $(A)$ where $l>1$. Then

$$
\limsup \frac{\log ^{[l]} \mathrm{T}_{h}^{-1} \mathrm{~T}_{\text {fog }}(\mathrm{r})}{\log ^{[l]} \mathrm{T}_{h}^{-1} \mathrm{~T}_{\mathrm{f}}\left(\exp \mathrm{r}^{\lambda_{g}}\right)} \leq \frac{\bar{\tau}_{\mathrm{g}} \cdot \rho_{\mathrm{h}}^{[\mathrm{ll}}(\mathrm{f})}{\lambda_{h}^{[l]}(\mathrm{f})} .
$$

Theorem 6 Let $\mathrm{f}$ be a meromorphic function, $\mathrm{g}$ and $\mathrm{h}$ be any two entire functions such that $\lambda_{\mathrm{h}}^{[\mathrm{ll}]}(\mathrm{g})>0, \rho_{\mathrm{h}}^{[\mathrm{ll}}(\mathrm{f})<\infty, \bar{\tau}_{\mathrm{g}}<\infty$ and $\mathrm{h}$ satisfy the Property (A) where $l>1$. Then

$$
\limsup \frac{\log ^{[l]} \mathrm{T}_{h}^{-1} \mathrm{~T}_{\text {fog }}(\mathrm{r})}{\log ^{[l]} \mathrm{T}_{h}^{-1} \mathrm{~T}_{g}\left(\exp \mathrm{r}^{\lambda_{g}}\right)} \leq \frac{\bar{\tau}_{g} \cdot \rho_{h}^{[l]}(\mathrm{f})}{\lambda_{h}^{[l]}(\mathrm{g})} .
$$

Theorem 7 Let $\mathrm{f}$ be meromorphic, $\mathrm{g}$ and $\mathrm{h}$ be any two entire functions such that $0<\lambda_{h}^{[l]}(\mathrm{f}) \leq \rho_{\mathrm{h}}^{[\mathrm{l}]}(\mathrm{f})<\infty, \tau_{\mathrm{g}}<\infty$ and $\mathrm{h}$ satisfy the Property $(A)$ where $l>1$. Then

$$
\liminf \frac{\log ^{[l]} \mathrm{T}_{h}^{-1} \mathrm{~T}_{\text {fog }}(r)}{\log ^{[l]} \mathrm{T}_{h}^{-1} \mathrm{~T}_{\mathrm{f}}\left(\exp \mathrm{r}^{\lambda_{g}}\right)} \leq \frac{\tau_{g} \cdot \rho_{h}^{[l]}(f)}{\lambda_{h}^{[l]}(f)} .
$$


Theorem 8 Let $\mathrm{f}$ be a meromorphic function, $\mathrm{g}$ and $\mathrm{h}$ be any two entire functions such that $\lambda_{\mathrm{h}}^{[\mathrm{ll}]}(\mathrm{g})>0, \rho_{\mathrm{h}}^{[\mathrm{l}]}(\mathrm{f})<\infty, \tau_{\mathrm{g}}<\infty$ and $\mathrm{h}$ satisfy the Property (A) where $l>1$. Then

$$
\liminf \frac{\log ^{[l]} \mathrm{T}_{h}^{-1} \mathrm{~T}_{f \circ g}(r)}{\log ^{[l]} \mathrm{T}_{h}^{-1} \mathrm{~T}_{g}\left(\exp \mathrm{r}^{\lambda_{g}}\right)} \leq \frac{\tau_{g} \cdot \rho_{h}^{[l]}(f)}{\lambda_{h}^{[l]}(g)} .
$$

Theorem 9 Let $\mathrm{f}$ be meromorphic and $\mathrm{g}, \mathrm{h}$ be any two entire functions such that (i) $0<\rho_{\mathrm{h}}^{[\mathrm{l}]}$ (f) $<\infty$, (ii) $\rho_{\mathrm{h}}^{[\mathrm{ll}]}$ (f) $=\rho_{\mathrm{g}}$, (iii) $\sigma_{\mathrm{g}}<\infty$, (iv) $0<\sigma_{\mathrm{h}}^{[\mathrm{ll}}$ (f) $<\infty$ and $\mathrm{h}$ satisfy the Property $(A)$ where $\mathrm{l}>1$. Then

$$
\liminf _{r \rightarrow \infty} \frac{\log ^{[l]} \mathrm{T}_{h}^{-1} \mathrm{~T}_{f \circ g}(r)}{\log ^{[l-1]} \mathrm{T}_{h}^{-1} \mathrm{~T}_{f}(\mathrm{r})} \leq \frac{\rho_{h}^{[l]}(f) \cdot \sigma_{g}}{\sigma_{h}^{[l]}(f)} .
$$

Proof. From (1), we get for all sufficiently large values of $r$ that

$$
\log ^{[l]} \mathrm{T}_{h}^{-1} \mathrm{~T}_{f \circ g}(r) \leqslant\left(\rho_{h}^{[l]}(f)+\varepsilon\right) \log M_{g}(r)+O(1) .
$$

Using Definition 2 we obtain from (3) for all sufficiently large values of $r$ that

$$
\log ^{[l]} \mathrm{T}_{h}^{-1} \mathrm{~T}_{f \circ g}(r) \leqslant\left(\rho_{h}^{[l]}(f)+\varepsilon\right)\left(\sigma_{g}+\varepsilon\right) \cdot r^{\rho_{g}}+O(1) .
$$

Now in view of condition (ii) we obtain from (4) for all sufficiently large values of $r$ that

$$
\log ^{[l]} T_{h}^{-1} T_{f \circ g}(r) \leqslant\left(\rho_{h}^{[l]}(f)+\varepsilon\right)\left(\sigma_{g}+\varepsilon\right) \cdot r_{h}^{\rho_{h}^{[l]}(f)}+O(1) .
$$

Again in view of Definition 6 we get for a sequence of values of $r$ tending to infinity that

$$
\log ^{[l-1]} T_{h}^{-1} T_{f}(r) \geq\left(\sigma_{h}^{[l]}(f)-\varepsilon\right) r_{h}^{\rho_{h}^{[l]}(f)} .
$$

Now from (5) and (6), it follows for a sequence of values of $r$ tending to infinity that

$$
\frac{\log ^{[l]} T_{h}^{-1} T_{f \circ g}(r)}{\log ^{[l-1]} T_{h}^{-1} T_{f}(r)} \leq \frac{\left(\rho_{h}^{[l]}(f)+\varepsilon\right)\left(\sigma_{g}+\varepsilon\right) \cdot r_{h}^{\rho_{h}^{[l]}(f)}+O(1)}{\left(\sigma_{h}^{[l]}(f)-\varepsilon\right) r^{\rho_{h}^{[l]}(f)}} .
$$

Since $\varepsilon(>0)$ is arbitrary, it follows from above that

$$
\liminf _{r \rightarrow \infty} \frac{\log ^{[l]} T_{h}^{-1} T_{f \circ g}(r)}{\log ^{[l-1]} T_{h}^{-1} T_{f}(r)} \leq \frac{\rho_{h}^{[l]}(f) \cdot \sigma_{g}}{\sigma_{h}^{[l]}(f)} .
$$


Hence the theorem follows.

Using the notion of lower type and relative lower type, we may state the following theorem without proof as it can be carried out in the line of Theorem 9:

Theorem 10 Let $\mathrm{f}$ be meromorphic and $\mathrm{g}, \mathrm{h}$ be any two entire functions such that (i) $0<\rho_{\mathrm{h}}^{[l]}$ (f) $<\infty$, (ii) $\rho_{\mathrm{h}}^{[\mathrm{l}]}$ (f) $=\rho_{\mathrm{g}}$, (iii) $\bar{\sigma}_{\mathrm{g}}<\infty$, (iv) $0<\bar{\sigma}_{\mathrm{h}}^{[\mathrm{l}]}$ (f) $<\infty$ and $\mathrm{h}$ satisfy the Property (A) where $\mathrm{l}>1$. Then

$$
\liminf _{r \rightarrow \infty} \frac{\log ^{[l]} T_{h}^{-1} T_{f \circ g}(r)}{\log ^{[l-1]} T_{h}^{-1} T_{f}(r)} \leq \frac{\rho_{h}^{[l]}(f) \cdot \bar{\sigma}_{g}}{\bar{\sigma}_{h}^{[l]}(f)} .
$$

Similarly using the notion of type and relative lower type one may state the following two theorems without their proofs because those can also be carried out in the line line of Theorem 9:

Theorem 11 Let $\mathrm{f}$ be meromorphic and $\mathrm{g}, \mathrm{h}$ be any two entire functions such that $(\mathfrak{i}) 0<\lambda_{h}^{[l]}(\mathrm{f}) \leq \rho_{\mathrm{h}}^{[\mathrm{ll}]}(\mathrm{f})<\infty$, (ii) $\rho_{\mathrm{h}}^{[\mathrm{l}]}(\mathrm{f})=\rho_{\mathrm{g}}$, (iii) $\sigma_{\mathrm{g}}<\infty$, (iv) $0<\bar{\sigma}_{\mathrm{h}}^{[\mathrm{ll}]}(\mathrm{f})<\infty$ and $\mathrm{h}$ satisfy the Property $(A)$ where $\mathrm{l}>1$. Then

$$
\liminf _{r \rightarrow \infty} \frac{\log ^{[l]} T_{h}^{-1} T_{f \circ g}(r)}{\log ^{[l-1]} T_{h}^{-1} T_{f}(r)} \leq \frac{\lambda_{h}^{[l]}(f) \cdot \sigma_{g}}{\bar{\sigma}_{h}^{[l]}(f)} .
$$

Theorem 12 Let $\mathrm{f}$ be meromorphic and $\mathrm{g}, \mathrm{h}$ be any two entire functions such that (i) $0<\rho_{\mathrm{h}}^{[l]}$ (f) $<\infty$, (ii) $\rho_{\mathrm{h}}^{[\mathrm{l}]}$ (f) $=\rho_{\mathrm{g}}$, (iii) $\sigma_{\mathrm{g}}<\infty$, (iv) $0<\bar{\sigma}_{\mathrm{h}}^{[\mathrm{l}]}$ (f) $<\infty$ and $\mathrm{h}$ satisfy the Property (A) where $\mathrm{l}>1$. Then

$$
\limsup _{r \rightarrow \infty} \frac{\log ^{[l]} \mathrm{T}_{h}^{-1} \mathrm{~T}_{f \circ g}(\mathrm{r})}{\log ^{[l-1]} \mathrm{T}_{h}^{-1} \mathrm{~T}_{\mathrm{f}}(\mathrm{r})} \leq \frac{\rho_{h}^{[l]}(\mathrm{f}) \cdot \sigma_{g}}{\bar{\sigma}_{h}^{[l]}(\mathrm{f})} .
$$

Similarly, using the concept of weak type and relative weak type, we may state next four theorems without their proofs as those can be carried out in the line of Theorem 9, Theorem 10, Theorem 11 and Theorem 12 respectively.

Theorem 13 Let $\mathrm{f}$ be meromorphic and $\mathrm{g}$, $\mathrm{h}$ be any two entire functions such that (i) $0<\lambda_{h}^{[l]}(f) \leq \rho_{h}^{[l]}(f)<\infty$, (ii) $\lambda_{h}^{[l]}$ (f) $=\lambda_{g}$, (iii) $\bar{\tau}_{g}<\infty$, (iv) $0<\bar{\tau}_{\mathrm{h}}^{[\mathrm{ll}}(\mathrm{f})<\infty$ and $\mathrm{h}$ satisfy the Property $(A)$ where $\mathrm{l}>1$. Then

$$
\liminf _{r \rightarrow \infty} \frac{\log ^{[l]} T_{h}^{-1} T_{f \circ g}(r)}{\log ^{[l-1]} T_{h}^{-1} T_{f}(r)} \leq \frac{\rho_{h}^{[[l]]}(f) \cdot \bar{\tau}_{g}}{\bar{\tau}_{h}^{[l]}(f)} .
$$


Theorem 14 Let $\mathrm{f}$ be meromorphic and $\mathrm{g}$, $\mathrm{h}$ be any two entire functions such that (i) $0<\lambda_{h}^{[l]}(\mathrm{f}) \leq \rho_{\mathrm{h}}^{[\mathrm{ll}}(\mathrm{f})<\infty$, (ii) $\lambda_{\mathrm{h}}^{[\mathrm{ll}}$ (f) $=\lambda_{\mathrm{g}}$, (iii) $\tau_{\mathrm{g}}<\infty$, (iv) $0<\tau_{\mathrm{h}}^{[\mathrm{l}]}(\mathrm{f})<\infty$ and $\mathrm{h}$ satisfy the Property $(A)$ where $\mathrm{l}>1$. Then

$$
\liminf _{r \rightarrow \infty} \frac{\log ^{[l]} T_{h}^{-1} T_{f \circ g}(r)}{\log ^{[l-1]} T_{h}^{-1} T_{f}(r)} \leq \frac{\rho_{h}^{[l]}(f) \cdot \tau_{g}}{\tau_{h}^{[l]}(f)} .
$$

Theorem 15 Let $\mathrm{f}$ be meromorphic and $\mathrm{g}, \mathrm{h}$ be any two entire functions such that (i) $0<\lambda_{h}^{[l]}\left(\right.$ f) $<\infty$, (ii) $\lambda_{h}^{[l]}\left(\right.$ f) $=\lambda_{g}$, (iii) $\bar{\tau}_{g}<\infty$, (iv) $0<\tau_{h}^{[l]}$ (f) $<\infty$ and $\mathrm{h}$ satisfy the Property (A) where $\mathrm{l}>1$. Then

$$
\liminf _{r \rightarrow \infty} \frac{\log ^{[l]} \mathrm{T}_{h}^{-1} \mathrm{~T}_{\text {fog }}(r)}{\log ^{[l-1]} \mathrm{T}_{h}^{-1} \mathrm{~T}_{f}(r)} \leq \frac{\lambda_{h}^{[l]}(f) \cdot \bar{\tau}_{g}}{\tau_{h}^{[l]}(f)} .
$$

Theorem 16 Let $\mathrm{f}$ be meromorphic and $\mathrm{g}, \mathrm{h}$ be any two entire functions such that (i) $0<\lambda_{h}^{[l]}$ (f) $\leq \rho_{h}^{[l]}(f)<\infty$, (ii) $\lambda_{h}^{[l]}$ (f) $=\lambda_{g}$, (iii) $\bar{\tau}_{g}<\infty$, (iv) $0<\tau_{\mathrm{h}}^{[\mathrm{l}]}(\mathrm{f})<\infty$ and $\mathrm{h}$ satisfy the Property $(A)$ where $\mathrm{l}>1$. Then

$$
\limsup _{r \rightarrow \infty} \frac{\log ^{[l]} \mathrm{T}_{h}^{-1} \mathrm{~T}_{f \circ g}(\mathrm{r})}{\log ^{[l-1]} \mathrm{T}_{h}^{-1} \mathrm{~T}_{\mathrm{f}}(\mathrm{r})} \leq \frac{\rho_{h}^{[l]}(\mathrm{f}) \cdot \bar{\tau}_{g}}{\tau_{h}^{[l]}(f)} .
$$

Theorem 17 Let $\mathrm{f}$ be meromorphic $\mathrm{g}, \mathrm{h}$ and $\mathrm{l}$ be any three entire functions such that $0<\bar{\sigma}_{h}^{[m]}(f \circ g) \leq \sigma_{h}^{[m]}(f \circ g)<\infty, 0<\bar{\sigma}_{l}^{[n]}(f) \leq \sigma_{l}^{[n]}(f)<\infty$ and $\rho_{\mathrm{h}}^{[\mathrm{m}]}(\mathrm{f} \circ \mathrm{g})=\rho_{\mathrm{l}}^{[\mathrm{n}]}(\mathrm{f})$ where $\mathrm{m}$ and $\mathrm{n}$ any positive integers $>1$. Then

$$
\begin{aligned}
\frac{\bar{\sigma}_{h}^{[m]}(f \circ g)}{\sigma[n]_{l}(f)} \leq \liminf _{r \rightarrow \infty} \frac{\log ^{[m-1]} T_{h}^{-1} T_{f \circ g}(r)}{\log ^{[n-1]} T_{l}^{-1} T_{f}(r)} \leq \frac{\bar{\sigma}_{h}^{[m]}(f \circ g)}{\bar{\sigma}_{l}^{[n]}(f)} \\
\quad \leq \limsup _{r \rightarrow \infty} \frac{\log ^{[m-1]} T_{h}^{-1} T_{f \circ g}(r)}{\log ^{[n-1]} T_{l}^{-1} T_{f}(r)} \leq \frac{\sigma_{h}^{[m]}(f \circ g)}{\bar{\sigma}_{l}^{[n]}(f)} .
\end{aligned}
$$

Proof. From the definition of $\sigma_{l}(f)$ and $\bar{\sigma}_{h}(f \circ g)$, we have for arbitrary positive $\varepsilon$ and for all sufficiently large values of $r$ that

$$
\log ^{[m-1]} \mathrm{T}_{h}^{-1} \mathrm{~T}_{\mathrm{f} \circ \mathrm{g}}(\mathrm{r}) \geqslant\left(\bar{\sigma}_{\mathrm{h}}^{[\mathrm{m}]}(\mathrm{f} \circ \mathrm{g})-\varepsilon\right) \mathrm{r}^{\mathrm{\rho}_{\mathrm{h}}^{[\mathrm{m}]}(\mathrm{f} \circ \mathrm{g})}
$$

and

$$
\log ^{[n-1]} T_{l}^{-1} T_{f}(r) \leq\left(\sigma_{l}^{[n]}(f)+\varepsilon\right) r^{\rho_{l}^{[n]}(f)}
$$


Now from $(7),(8)$ and the condition $\rho_{h}^{[m]}(f \circ g)=\rho_{l}^{[n]}(f)$, it follows for all large values of $r$ that,

$$
\frac{\log ^{[m-1]} T_{h}^{-1} T_{f \circ g}(r)}{\log ^{[n-1]} T_{l}^{-1} T_{f}(r)} \geqslant \frac{\left(\bar{\sigma}_{h}^{[m]}(f \circ g)-\varepsilon\right)}{\left(\sigma_{l}^{[n]}(f)+\varepsilon\right)} .
$$

As $\mathcal{\varepsilon}(>0)$ is arbitrary, we obtain that

$$
\liminf _{r \rightarrow \infty} \frac{\log ^{[m-1]} T_{h}^{-1} T_{f \circ g}(r)}{\log ^{[n-1]} T_{l}^{-1} T_{f}(r)} \geqslant \frac{\bar{\sigma}_{h}^{[m]}(f \circ g)}{\sigma_{l}^{[n]}(f)} .
$$

Again for a sequence of values of $r$ tending to infinity,

$$
\log ^{[m-1]} T_{h}^{-1} T_{f \circ g}(r) \leq\left(\bar{\sigma}_{h}^{[m]}(f \circ g)+\varepsilon\right) r^{\rho_{h}^{[m]}(f \circ g)}
$$

and for all sufficiently large values of $r$,

$$
\log ^{[n-1]} T_{l}^{-1} T_{f}(r) \geqslant\left(\bar{\sigma}_{l}^{[n]}(f)-\varepsilon\right) r^{\rho_{l}^{[n]}(f)} .
$$

Combining the condition $\rho_{h}(f \circ g)=\rho_{l}(f),(10)$ and (11) we get for a sequence of values of $r$ tending to infinity that

$$
\frac{\log ^{[m-1]} T_{h}^{-1} T_{f \circ g}(r)}{\log ^{[n-1]} T_{l}^{-1} T_{f}(r)} \leq \frac{\left(\bar{\sigma}_{h}^{[m]}(f \circ g)+\varepsilon\right)}{\left(\bar{\sigma}_{l}^{[n]}(f)-\varepsilon\right)} .
$$

Since $\varepsilon(>0)$ is arbitrary, it follows that

$$
\liminf _{r \rightarrow \infty} \frac{\log ^{[m-1]} \mathrm{T}_{h}^{-1} \mathrm{~T}_{f \circ g}(r)}{\log ^{[n-1]} \mathrm{T}_{l}^{-1} \mathrm{~T}_{\mathrm{f}}(\mathrm{r})} \leq \frac{{\overline{\sigma_{h}}}_{\mathrm{m}]}(\mathrm{f} \circ \mathrm{g})}{\bar{\sigma}_{l}^{[\mathrm{n}]}(\mathrm{f})} .
$$

Also for a sequence of values of $r$ tending to infinity that

$$
\log ^{[n-1]} T_{l}^{-1} T_{f}(r) \leq\left(\bar{\sigma}_{l}^{[n]}(f)+\varepsilon\right) r^{\rho_{l}^{[n]}(f)} .
$$

Now from (7), (13) and the condition $\rho_{\mathrm{h}}(\mathrm{f} \circ \mathrm{g})=\rho_{\mathrm{l}}(\mathrm{f})$, we obtain for a sequence of values of $r$ tending to infinity that

$$
\frac{\log ^{[m-1]} T_{h}^{-1} T_{f \circ g}(r)}{\log ^{[n-1]} T_{l}^{-1} T_{f}(r)} \geq \frac{\left(\bar{\sigma}_{h}^{[m]}(f \circ g)-\varepsilon\right)}{\left(\bar{\sigma}_{l}^{[n]}(f)+\varepsilon\right)} .
$$


As $\varepsilon(>0)$ is arbitrary, we get from above that

$$
\limsup _{r \rightarrow \infty} \frac{\log ^{[m-1]} \mathrm{T}_{h}^{-1} \mathrm{~T}_{\text {fog }}(\mathrm{r})}{\log ^{[\mathrm{n}-1]} \mathrm{T}_{l}^{-1} \mathrm{~T}_{\mathrm{f}}(\mathrm{r})} \geq \frac{{\overline{\sigma_{h}}}_{\mathrm{m}]}^{[\mathrm{f}}(\mathrm{f} \circ \mathrm{g})}{\bar{\sigma}_{l}^{[\mathrm{n}]}(\mathrm{f})} .
$$

Also for all sufficiently large values of $r$,

$$
\log ^{[n-1]} T_{h}^{-1} T_{f \circ g}(r) \leq\left(\bar{\sigma}_{h}^{[m]}(f \circ g)+\varepsilon\right) r^{\rho_{h}^{[m]}(f \circ g)} .
$$

As the condition $\rho_{h}(f \circ g)=\rho_{l}(f)$, it follows from (11) and (15) for all sufficiently large values of $r$ that

$$
\frac{\log ^{[m-1]} T_{h}^{-1} T_{f \circ g}(r)}{\log ^{[n-1]} T_{l}^{-1} T_{f}(r)} \leq \frac{\left(\bar{\sigma}_{h}^{[m]}(f \circ g)+\varepsilon\right)}{\left(\bar{\sigma}_{l}^{[n]}(f)-\varepsilon\right)} .
$$

Since $\varepsilon(>0)$ is arbitrary, we obtain that

$$
\limsup _{r \rightarrow \infty} \frac{\log ^{[m-1]} \mathrm{T}_{h}^{-1} \mathrm{~T}_{f \circ g}(r)}{\log ^{[n-1]} \mathrm{T}_{l}^{-1} \mathrm{~T}_{\mathrm{f}}(\mathrm{r})} \leq \frac{\bar{\sigma}_{h}^{[m]}(\mathrm{f} \circ \mathrm{g})}{\bar{\sigma}_{l}^{[n]}(f)} .
$$

Thus the theorem follows from (9), (12), (14) and (16).

The following theorem can be proved in the line of Theorem 17 and so the proof is omitted.

Theorem 18 Let $\mathrm{f}$ be meromorphic, $\mathrm{g}, \mathrm{h}$ and $\mathrm{k}$ be any three entire functions such that $0<\bar{\sigma}_{\mathrm{h}}^{[\mathrm{m}]}(\mathrm{f} \circ \mathrm{g}) \leq \sigma_{\mathrm{h}}^{[\mathrm{m}]}(\mathrm{f} \circ \mathrm{g})<\infty, 0<\bar{\sigma}_{\mathrm{k}}^{[\mathrm{n}]}(\mathrm{g}) \leq \sigma_{\mathrm{k}}^{[\mathrm{n}]}(\mathrm{g})<\infty$ and $\rho_{\mathrm{h}}^{[\mathrm{m}]}(\mathrm{f} \circ \mathrm{g})=\rho_{\mathrm{k}}^{[\mathrm{n}]}(\mathrm{g})$ where $\min \{\mathrm{m}, \mathrm{n}\}>1$. Then

$$
\begin{aligned}
\frac{\bar{\sigma}_{h}^{[m]}(f \circ g)}{\sigma_{k}^{[n]}(g)} & \leq \liminf _{r \rightarrow \infty} \frac{\log ^{[m-1]} T_{h}^{-1} T_{f \circ g}(r)}{\log ^{[n-1]} T_{k}^{-1} T_{g}(r)} \leq \frac{\bar{\sigma}_{h}^{[m]}(f \circ g)}{\bar{\sigma}_{k}^{[n]}(g)} \\
& \leq \limsup _{r \rightarrow \infty} \frac{\log ^{[m-1]} T_{h}^{-1} T_{f \circ g}(r)}{\log ^{[n-1]} T_{k}^{-1} T_{g}(r)} \leq \frac{\sigma_{h}^{[m]}(f \circ g)}{\bar{\sigma}_{k}^{[n]}(g)} .
\end{aligned}
$$

Theorem 19 Let $\mathrm{f}$ be meromorphic $\mathrm{g}, \mathrm{h}$ and $\mathrm{l}$ be any three entire functions such that $0<\sigma_{\mathrm{h}}^{[\mathrm{m}]}(\mathrm{f} \circ \mathrm{g})<\infty, 0<\sigma_{\mathrm{l}}^{[\mathrm{n}]}(\mathrm{f})<\infty$ and $\rho_{\mathrm{h}}^{[\mathrm{m}]}(\mathrm{f} \circ \mathrm{g})=\rho_{\mathrm{l}}^{[\mathrm{n}]}(\mathrm{f})$ where $\mathrm{m}$ and $\mathrm{n}$ are any positive integers with $\mathrm{m}>1$ and $\mathrm{n}>1$ respectively. Then

$$
\liminf _{r \rightarrow \infty} \frac{\log ^{[m-1]} T_{h}^{-1} T_{f \circ g}(r)}{\log ^{[n-1]} T_{l}^{-1} T_{f}(r)} \leq \frac{\sigma_{h}^{[m]}(f \circ g)}{\sigma_{l}^{[n]}(f)} \leq \limsup _{r \rightarrow \infty} \frac{\log ^{[m-1]} T_{h}^{-1} T_{f \circ g}(r)}{\log ^{[n-1]} T_{l}^{-1} T_{f}(r)} .
$$


Proof. From the definition of $\sigma_{l}^{[n]}(f)$, we get for a sequence of values of $r$ tending to infinity that

$$
\log ^{[n-1]} T_{l}^{-1} T_{f}(r) \geqslant\left(\sigma_{l}^{[n]}(f)-\varepsilon\right) r^{\rho_{l}^{[n]}(f)} .
$$

Now from $(15),(17)$ and the condition $\rho_{h}^{[m]}(f \circ g)=\rho_{l}^{[b]}(f)$, it follows for a sequence of values of $r$ tending to infinity that

$$
\frac{\log ^{[m-1]} T_{h}^{-1} T_{f \circ g}(r)}{\log ^{[n-1]} T_{l}^{-1} T_{f}(r)} \leq \frac{\left(\sigma_{h}^{[m]}(f \circ g)+\varepsilon\right)}{\left(\sigma_{l}^{[n]}(f)-\varepsilon\right)} .
$$

As $\mathcal{E}(>0)$ is arbitrary, we obtain that

$$
\liminf _{r \rightarrow \infty} \frac{\log ^{[m-1]} T_{h}^{-1} T_{f \circ g}(r)}{\log ^{[n-1]} T_{l}^{-1} T_{f}(r)} \leq \frac{\sigma_{h}^{[m]}(f \circ g)}{\sigma_{l}^{[n]}(f)} .
$$

Again for a sequence of values of $r$ tending to infinity,

$$
\log ^{[m-1]} T_{h}^{-1} T_{f \circ g}(r) \geqslant\left(\sigma_{h}^{[m]}(f \circ g)-\varepsilon\right) r^{\rho_{h}^{[m]}(f \circ g)} .
$$

So combining the condition $\rho_{h}(f \circ g)=\rho_{l}(f),(8)$ and (19), we get for a sequence of values of $r$ tending to infinity that

$$
\frac{\log ^{[m-1]} T_{h}^{-1} T_{f \circ g}(r)}{\log ^{[n-1]} T_{l}^{-1} T_{f}(r)} \geqslant \frac{\left(\sigma_{h}^{[m]}(f \circ g)-\varepsilon\right)}{\left(\sigma_{l}^{[n]}(f)+\varepsilon\right)} .
$$

Since $\varepsilon(>0)$ is arbitrary, it follows that

$$
\limsup _{r \rightarrow \infty} \frac{\log ^{[m-1]} \mathrm{T}_{h}^{-1} \mathrm{~T}_{\mathrm{f} \circ \mathrm{g}}(\mathrm{r})}{\log ^{[\mathrm{n}-1]} \mathrm{T}_{l}^{-1} \mathrm{~T}_{\mathrm{f}}(\mathrm{r})} \geqslant \frac{\sigma_{h}^{[m]}(\mathrm{f} \circ \mathrm{g})}{\sigma_{l}^{[n]}(f)} .
$$

Thus the theorem follows from (18) and (20).

The following theorem can be carried out in the line of Theorem 19 and therefore we omit its proof.

Theorem 20 Let $\mathrm{f}$ be meromorphic, $\mathrm{g}, \mathrm{h}$ and $\mathrm{k}$ be any three entire functions such that $0<\sigma_{\mathrm{h}}^{[\mathrm{m}]}(\mathrm{f} \circ \mathrm{g})<\infty, 0<\sigma_{\mathrm{k}}^{[\mathrm{n}]}(\mathrm{g})<\infty$ and $\rho_{\mathrm{h}}^{[\mathrm{m}]}(\mathrm{f} \circ \mathrm{g})=\rho_{\mathrm{k}}^{[\mathrm{n}]}(\mathrm{g})$ where $\mathrm{m}$ and $\mathrm{n}$ are any positive integers $>1$. Then

$$
\liminf _{r \rightarrow \infty} \frac{\log ^{[m-1]} T_{h}^{-1} T_{f \circ g}(r)}{\log ^{[n-1]} T_{k}^{-1} T_{g}(r)} \leq \frac{\sigma_{h}^{[m]}(f \circ g)}{\sigma_{k}^{[n]}(g)} \leq \limsup _{r \rightarrow \infty} \frac{\log ^{[m-1]} T_{h}^{-1} T_{f \circ g}(r)}{\log ^{[n-1]} T_{k}^{-1} T_{g}(r)} .
$$


The following theorem is a natural consequence of Theorem 17 and Theorem 19.

Theorem 21 Let $\mathrm{f}$ be meromorphic $\mathrm{g}, \mathrm{h}$ and $\mathrm{l}$ be any three entire functions such that $0<\bar{\sigma}_{\mathrm{h}}^{[\mathrm{m}]}(\mathrm{f} \circ \mathrm{g}) \leq \sigma_{\mathrm{h}}^{[\mathrm{m}]}(\mathrm{f} \circ \mathrm{g})<\infty, 0<\bar{\sigma}_{\mathrm{l}}^{[\mathrm{n}]}(\mathrm{f}) \leq \sigma_{\mathrm{l}}^{[\mathrm{n}]}(\mathrm{f})<\infty$ and $\rho_{\mathrm{h}}^{[\mathrm{m}]}(\mathrm{f} \circ \mathrm{g})=\rho_{\mathrm{l}}^{[\mathrm{n}]}(\mathrm{f})$ where $\mathrm{m}$ and $\mathrm{n}$ are any positive integers with $\mathrm{m}>1$ and $\mathrm{n}>1$ respectively. Then

$$
\begin{aligned}
& \liminf _{r \rightarrow \infty} \frac{\log ^{[m-1]} T_{h}^{-1} T_{f \circ g}(r)}{\log ^{[n-1]} T_{l}^{-1} T_{f}(r)} \leq \min \left\{\frac{\bar{\sigma}_{h}^{[m]}(f \circ g)}{\bar{\sigma}_{l}^{[n]}(f)}, \frac{\sigma_{h}^{[m]}(f \circ g)}{\sigma_{l}^{[n]}(f)}\right\} \\
& \quad \leq \max \left\{\frac{\bar{\sigma}_{h}^{[m]}(f \circ g)}{\bar{\sigma}_{l}^{[n]}(f)}, \frac{\sigma_{h}^{[m]}(f \circ g)}{\sigma_{l}^{[n]}(f)}\right\} \leq \limsup _{r \rightarrow \infty} \frac{\log ^{[m-1]} T_{h}^{-1} T_{f \circ g}(r)}{\log ^{[n-1]} T_{l}^{-1} T_{f}(r)} .
\end{aligned}
$$

The proof is omitted.

Analogously one may state the following theorem without its proof as it is also a natural consequence of Theorem 18 and Theorem 20 .

Theorem 22 Let $\mathrm{f}$ be meromorphic, $\mathrm{g}, \mathrm{h}$ and $\mathrm{k}$ be any three entire functions such that $0<\bar{\sigma}_{\mathrm{h}}^{[\mathrm{m}]}(\mathrm{f} \circ \mathrm{g}) \leq \sigma_{\mathrm{h}}^{[\mathrm{m}]}(\mathrm{f} \circ \mathrm{g})<\infty, 0<\bar{\sigma}_{\mathrm{k}}^{[\mathrm{n}]}(\mathrm{g}) \leq \sigma_{\mathrm{k}}^{[\mathrm{n}]}(\mathrm{g})<\infty$ and $\rho_{\mathrm{h}}^{[\mathrm{m}]}(\mathrm{f} \circ \mathrm{g})=\rho_{\mathrm{k}}^{[\mathrm{n}]}(\mathrm{g})$ where $\mathrm{m}$ and $\mathrm{n}$ are any positive integers $>1$. Then

$$
\begin{aligned}
& \liminf _{r \rightarrow \infty} \frac{\log ^{[m-1]} T_{h}^{-1} T_{f \circ g}(r)}{\log ^{[n-1]} T_{k}^{-1} T_{g}(r)} \leq \min \left\{\frac{\bar{\sigma}_{h}^{[m]}(f \circ g)}{\bar{\sigma}_{k}^{[n]}(g)}, \frac{\sigma_{h}^{[m]}(f \circ g)}{\sigma_{k}^{[n]}(g)}\right\} \\
& \leq \max \left\{\frac{\bar{\sigma}_{h}^{[m]}(f \circ g)}{\bar{\sigma}_{k}^{[n]}(g)}, \frac{\sigma_{h}^{[m]}(f \circ g)}{\sigma_{k}^{[n]}(g)}\right\} \leq \limsup _{r \rightarrow \infty} \frac{\log ^{[m-1]} T_{h}^{-1} T_{f \circ g}(r)}{\log ^{[n-1]} T_{k}^{-1} T_{g}(r)} .
\end{aligned}
$$

In the same way, using the concept of relative weak type, we may state next two theorems without their proofs as those can be carried out in the line of Theorem 17 and Theorem 19 respectively.

Theorem 23 Let $\mathrm{f}$ be meromorphic $\mathrm{g}, \mathrm{h}$ and $\mathrm{l}$ be any three entire functions such that $0<\tau_{\mathrm{h}}^{[\mathrm{m}]}(\mathrm{f} \circ \mathrm{g}) \leq \bar{\tau}_{\mathrm{h}}^{[\mathrm{m}]}(\mathrm{f} \circ \mathrm{g})<\infty, 0<\tau_{\mathrm{l}}^{[\mathrm{n}]}(\mathrm{f}) \leq \bar{\tau}_{\mathrm{l}}^{[\mathrm{n}]}(\mathrm{f})<\infty$ and $\lambda_{h}^{[m]}(f \circ g)=\lambda_{l}^{[n]}(f)$ where $m$ and $n$ any positive integers $>1$. Then

$$
\begin{aligned}
\frac{\tau_{h}^{[m]}(f \circ g)}{\bar{\tau}_{l}^{[n]}(f)} & \leq \liminf _{r \rightarrow \infty} \frac{\log ^{[m-1]} T_{h}^{-1} T_{f \circ g}(r)}{\log ^{[n-1]} T_{l}^{-1} T_{f}(r)} \leq \frac{\tau_{h}^{[m]}(f \circ g)}{\tau_{l}^{[n]}(f)} \\
& \leq \limsup _{r \rightarrow \infty} \frac{\log ^{[m-1]} T_{h}^{-1} T_{f \circ g}(r)}{\log ^{[n-1]} T_{l}^{-1} T_{f}(r)} \leq \frac{\bar{\tau}_{h}^{[m]}(f \circ g)}{\tau_{l}^{[n]}(f)} .
\end{aligned}
$$


Theorem 24 Let $\mathrm{f}$ be meromorphic $\mathrm{g}, \mathrm{h}$ and $\mathrm{l}$ be any three entire functions such that $0<\bar{\tau}_{\mathrm{h}}^{[\mathrm{m}]}(\mathrm{f} \circ \mathrm{g})<\infty, 0<\bar{\tau}_{\mathrm{l}}^{[\mathrm{n}]}(\mathrm{f})<\infty$ and $\lambda_{\mathrm{h}}^{[\mathrm{m}]}(\mathrm{f} \circ \mathrm{g})=\lambda_{\mathrm{l}}^{[\mathrm{n}]}(\mathrm{f})$ where $\mathrm{m}$ and $\mathrm{n}$ are any positive integers with $\mathrm{m}>1$ and $\mathrm{n}>1$ respectively. Then

$$
\liminf _{r \rightarrow \infty} \frac{\log ^{[m-1]} T_{h}^{-1} T_{f \circ g}(r)}{\log ^{[n-1]} T_{l}^{-1} T_{f}(r)} \leq \frac{\bar{\tau}_{h}^{[m]}(f \circ g)}{\bar{\tau}_{l}^{[n]}(f)} \leq \limsup _{r \rightarrow \infty} \frac{\log ^{[m-1]} T_{h}^{-1} T_{f \circ g}(r)}{\log ^{[n-1]} T_{l}^{-1} T_{f}(r)} .
$$

The following theorem is a natural consequence of Theorem 23 and Theorem 24:

Theorem 25 Let $\mathrm{f}$ be meromorphic $\mathrm{g}, \mathrm{h}$ and $\mathrm{l}$ be any three entire functions such that $0<\tau_{h}^{[m]}(f \circ g) \leq \bar{\tau}_{h}^{[m]}(f \circ g)<\infty, 0<\tau_{l}^{[n]}(f) \leq \bar{\tau}_{l}^{[n]}(f)<\infty$ and $\lambda_{h}^{[m]}(f \circ g)=\lambda_{l}^{[n]}(f)$ where $m$ and $n$ any positive integers $>1$. Then

$$
\begin{aligned}
& \liminf _{r \rightarrow \infty} \frac{\log ^{[m-1]} T_{h}^{-1} T_{f \circ g}(r)}{\log ^{[n-1]} T_{l}^{-1} T_{f}(r)} \leq \min \left\{\frac{\bar{\tau}_{h}^{[m]}(f \circ g)}{\bar{\tau}_{l}^{[n]}(f)}, \frac{\tau_{h}^{[m]}(f \circ g)}{\tau_{l}^{[n]}(f)}\right\} \\
& \leq \max \left\{\frac{\bar{\tau}_{h}^{[m]}(f \circ g)}{\bar{\tau}_{l}^{[n]}(f)}, \frac{\tau_{h}^{[m]}(f \circ g)}{\tau_{l}^{[n]}(f)}\right\} \leq \limsup _{r \rightarrow \infty} \frac{\log ^{[m-1]} T_{h}^{-1} T_{f \circ g}(r)}{\log ^{[n-1]} T_{l}^{-1} T_{f}(r)} .
\end{aligned}
$$

The following two theorems can be proved in the line of Theorem 23 and Theorem 24 respectively and therefore their proofs are omitted.

Theorem 26 Let $\mathrm{f}$ be meromorphic, $\mathrm{g}, \mathrm{h}$ and $\mathrm{k}$ be any three entire functions such that $0<\tau_{\mathrm{h}}^{[\mathrm{m}]}(\mathrm{f} \circ \mathrm{g}) \leq \bar{\tau}_{\mathrm{h}}^{[\mathrm{m}]}(\mathrm{f} \circ \mathrm{g})<\infty, 0<\tau_{\mathrm{k}}^{[\mathrm{n}]}(\mathrm{g}) \leq \bar{\tau}_{\mathrm{k}}^{[\mathrm{n}]}(\mathrm{g})<\infty$ and $\lambda_{\mathrm{h}}^{[\mathrm{m}]}(\mathrm{f} \circ \mathrm{g})=\lambda_{\mathrm{k}}^{[\mathrm{n}]}(\mathrm{g})$ where $\mathrm{m}$ and $\mathrm{n}$ are any positive integers $>1$. Then

$$
\begin{aligned}
\frac{\tau_{h}^{[m]}(f \circ g)}{\bar{\tau}_{k}^{[n]}(g)} & \leq \liminf _{r \rightarrow \infty} \frac{\log ^{[m-1]} T_{h}^{-1} T_{f \circ g}(r)}{\log ^{[m-1]} T_{k}^{-1} T_{g}(r)} \leq \frac{\tau_{h}^{[m]}(f \circ g)}{\tau_{k}^{[n]}(g)} \\
& \leq \limsup _{r \rightarrow \infty} \frac{\log ^{[m-1]} T_{h}^{-1} T_{f \circ g}(r)}{\log ^{[m-1]} T_{k}^{-1} T_{g}(r)} \leq \frac{\bar{\tau}_{h}^{[m]}(f \circ g)}{\tau_{k}^{[n]}(g)} .
\end{aligned}
$$

Theorem 27 Let $\mathrm{f}$ be meromorphic, $\mathrm{g}, \mathrm{h}$ and $\mathrm{k}$ be any three entire functions such that $0<\bar{\tau}_{\mathrm{h}}^{[\mathrm{m}]}(\mathrm{f} \circ \mathrm{g})<\infty, 0<\bar{\tau}_{\mathrm{k}}^{[\mathrm{n}]}(\mathrm{g})<\infty$ and $\lambda_{\mathrm{h}}^{[\mathrm{m}]}(\mathrm{f} \circ \mathrm{g})=\lambda_{\mathrm{k}}^{[\mathrm{n}]}(\mathrm{g})$ where $\mathrm{m}$ and $\mathrm{n}$ any positive integers $>1$. Then

$$
\liminf _{r \rightarrow \infty} \frac{\log ^{[m-1]} \mathrm{T}_{h}^{-1} \mathrm{~T}_{f \circ g}(r)}{\log ^{[m-1]} \mathrm{T}_{k}^{-1} \mathrm{~T}_{g}(r)} \leq \frac{\bar{\tau}_{h}^{[m]}(f \circ g)}{\bar{\tau}_{k}^{[n]}(g)} \leq \limsup _{r \rightarrow \infty} \frac{\log ^{[m-1]} \mathrm{T}_{h}^{-1} \mathrm{~T}_{f \circ g}(r)}{\log ^{[m-1]} \mathrm{T}_{k}^{-1} \mathrm{~T}_{g}(\mathrm{r})} .
$$


The following theorem is a natural consequence of Theorem 26 and Theorem 27.

Theorem 28 Let $\mathrm{f}$ be meromorphic, $\mathrm{g}, \mathrm{h}$ and $\mathrm{k}$ be any three entire functions such that $0<\tau_{\mathrm{h}}^{[\mathrm{m}]}(\mathrm{f} \circ \mathrm{g}) \leq \bar{\tau}_{\mathrm{h}}^{[\mathrm{m}]}(\mathrm{f} \circ \mathrm{g})<\infty, 0<\tau_{\mathrm{k}}^{[\mathrm{n}]}(\mathrm{g}) \leq \bar{\tau}_{\mathrm{k}}^{[\mathrm{n}]}(\mathrm{g})<\infty$ and $\lambda_{\mathrm{h}}^{[\mathrm{m}]}(\mathrm{f} \circ \mathrm{g})=\lambda_{\mathrm{k}}^{[\mathrm{n}]}(\mathrm{g})$ where $\mathrm{m}$ and $\mathrm{n}$ are any positive integers $>1$. Then

$$
\begin{aligned}
& \liminf _{r \rightarrow \infty} \frac{\log ^{[m-1]} \mathrm{T}_{h}^{-1} \mathrm{~T}_{\mathrm{f} \circ \mathrm{g}}(\mathrm{r})}{\log ^{[\mathrm{m}-1]} \mathrm{T}_{\mathrm{k}}^{-1} \mathrm{~T}_{\mathrm{g}}(\mathrm{r})} \leq \min \left\{\frac{\bar{\tau}_{\mathrm{h}}^{[\mathrm{m}]}(\mathrm{f} \circ \mathrm{g})}{\bar{\tau}_{\mathrm{k}}^{[\mathrm{n}]}(\mathrm{g})}, \frac{\tau_{\mathrm{h}}^{[\mathrm{m}]}(\mathrm{f} \circ \mathrm{g})}{\tau_{\mathrm{k}}^{[\mathrm{n}]}(\mathrm{g})}\right\} \\
& \leq \max \left\{\frac{\bar{\tau}_{h}^{[m]}(\mathrm{f} \circ \mathrm{g})}{\bar{\tau}_{\mathrm{k}}^{[\mathrm{n}]}(\mathrm{g})}, \frac{\tau_{\mathrm{h}}^{[\mathrm{m}]}(\mathrm{f} \circ \mathrm{g})}{\tau_{\mathrm{k}}^{[\mathrm{n}]}(\mathrm{g})}\right\} \leq \limsup _{\mathrm{r} \rightarrow \infty} \frac{\log ^{[\mathrm{m}-1]} \mathrm{T}_{h}^{-1} \mathrm{~T}_{\mathrm{f} \circ \mathrm{g}}(\mathrm{r})}{\log ^{[\mathrm{m}-1]} \mathrm{T}_{\mathrm{k}}^{-1} \mathrm{~T}_{\mathrm{g}}(\mathrm{r})} .
\end{aligned}
$$

Theorem 29 Let $\mathrm{f}$ be meromorphic, $\mathrm{g}$ and $\mathrm{h}$ be any two entire functions such that $0<\lambda_{h}^{[l]}(f) \leq \rho_{h}^{[l]}(f)<\rho_{g} \leq \infty$ and $\sigma_{h}^{[l]}(f)<\infty$ where $l>1$. Then

$$
\limsup _{r \rightarrow \infty} \frac{\log ^{[l]} \mathrm{T}_{h}^{-1} \mathrm{~T}_{\text {fog }}(r)}{\log ^{[l-1]} \mathrm{T}_{h}^{-1} \mathrm{~T}_{f}(\mathrm{r})} \geq \frac{\lambda_{h}^{[l]}(f)}{\sigma_{h}^{[l]}(f)} .
$$

Proof. Since $\rho_{h}^{[l]}(f)<\rho_{g}$ and $T_{h}^{-1}(r)$ is a increasing function of $r$, we get from Lemma 2 for a sequence of values of $r$ tending to infinity that

$$
\begin{aligned}
\log ^{[l]} \mathrm{T}_{h}^{-1} \mathrm{~T}_{\text {fog }}(r) & \geq \log ^{[l]} \mathrm{T}_{h}^{-1} \mathrm{~T}_{f}\left(\exp \left(\mathrm{r}^{\mu}\right)\right) \\
\text { i.e., } \log ^{[l]} \mathrm{T}_{h}^{-1} \mathrm{~T}_{f \circ g}(r & \geq\left(\lambda_{h}^{[l]}(f)-\varepsilon\right) \cdot r^{\mu} \\
\text { i.e., } \log ^{[l]} \mathrm{T}_{h}^{-1} \mathrm{~T}_{\text {fog }}(r) & \geq\left(\lambda_{h}^{[l]}(f)-\varepsilon\right) \cdot r^{[l]}(f)
\end{aligned}
$$

Again in view of Definition 6, we get for all sufficiently large values of $r$ that

$$
\log ^{[l-1]} \mathrm{T}_{h}^{-1} \mathrm{~T}_{\mathrm{f}}(\mathrm{r}) \leq\left(\sigma_{h}^{[l]}(f)+\varepsilon\right) r_{h}^{\rho_{h}^{[l]}(f)} .
$$

Now from (21) and (22), it follows for a sequence of values of $r$ tending to infinity that

$$
\frac{\log ^{[l]} \mathrm{T}_{h}^{-1} T_{f \circ g}(r)}{\log ^{[l-1]} T_{h}^{-1} T_{f}(r)} \geq \frac{\left(\lambda_{h}^{[l]}(f)-\varepsilon\right) \cdot r^{\rho_{h}^{[l]}(f)}}{\left(\sigma_{h}^{[l]}(f)+\varepsilon\right) r_{h}^{\rho_{h}^{[l]}(f)}} .
$$

Since $\varepsilon(>0)$ is arbitrary, it follows from above that

$$
\limsup _{r \rightarrow \infty} \frac{\log ^{[l]} \mathrm{T}_{h}^{-1} \mathrm{~T}_{\text {fog }}(r)}{\log ^{[l-1]} \mathrm{T}_{h}^{-1} \mathrm{~T}_{f}(\mathrm{r})} \geq \frac{\lambda_{h}^{[l]}(f)}{\sigma_{h}^{[l]}(f)} .
$$


Thus the theorem follows.

Now we state the following theorem without its proof as it can be carried out in the line of Theorem 29 and with the help of Definition 7:

Theorem 30 Let $\mathrm{f}$ be meromorphic, $\mathrm{g}$ and $\mathrm{h}$ be any two entire functions such that $0<\lambda_{h}^{[l]}(f)<\rho_{g} \leq \infty$ and $\bar{\tau}_{h}^{[l]}(f)<\infty$ where $l>1$. Then

$$
\limsup _{r \rightarrow \infty} \frac{\log ^{[l]} \mathrm{T}_{h}^{-1} \mathrm{~T}_{\text {fog }}(r)}{\log ^{[l-1]} \mathrm{T}_{h}^{-1} \mathrm{~T}_{f}(r)} \geq \frac{\lambda_{h}^{[l]}(f)}{\bar{\tau}_{h}^{[l]}(f)} .
$$

\section{Acknowledgments}

The authors are thankful to referee for his/her valuable suggestions towards the improvement of the paper.

\section{References}

[1] L. Bernal, Crecimiento relativo de funciones enteras. Contribucion al estudio de lasfunciones enteras con índice exponencial finito, Doctoral Dissertation, University of Seville, Spain, 1984.

[2] L. Bernal, Orden relative de crecimiento de funciones enteras, Collect. Math., Vol. 39 (1988), 209-229.

[3] W. Bergweiler, On the Nevanlinna characteristic of a composite function, Complex Var. Elliptic Equ., 10 (1988), 225-236.

[4] W. Bergweiler, On the growth rate of composite meromorphic functions, Complex Var. Elliptic Equ., 14 (1990), 187-196.

[5] D. Banerjee, S. Jana, Meromorphic functions of relative order ( $p, q)$, Soochow Journal of Mathematics, 33 (3) (2007), 343-357.

[6] S. K. Datta, A. Jha, On the weak type of meromorphic functions, Int. Math. Forum, 4 (12) (2009), 569-579.

[7] S. K. Datta, T. Biswas, C. Biswas: Measure of growth ratios of composite entire and meromorphic functions with a focus on relative order, International J. of Math. Sci. E Engg. Appls. (IJMSEA), 8 (2014), 207-218. 
[8] W. K. Hayman, Meromorphic Functions, The Clarendon Press, Oxford, 1964.

[9] B. K. Lahiri, D. Banerjee, Relative order of entire and meromorphic functions, Proc. Nat. Acad. Sci. India Ser. A., 69 (A) 3, (1999), 339-354.

[10] D. Sato, On the rate of growth of entire functions of fast growth, Bull. Amer. Math. Soc., 69 (1963), 411-414.

[11] E. C. Titchmarsh, The theory of functions, 2nd ed. Oxford University Press, Oxford, 1968.

[12] G. Valiron, Lectures on the general theory of integral functions, Chelsea Publishing Company, 1949.

Received: April 4, 2016 